\title{
PERFIL EPIDEMIOLÓGICO DO CÂNCER DE PRÓSTATA NO BRASIL: RETRATO DE UMA DÉCADA
}

\section{EPIDEMIOLOGICAL PROFILE OF PROSTATE CANCER IN BRAZIL: A DECADE PORTRAIT}

Lívia Silva De Paula Faria*, Pedro Caldas Pereira, André Luismorelli Lustosa, lapunira Catarina Sant'anna Aragão, Felipe Matheus Sant'anna Aragão, Marcos Guimarães De Souza Cunha.

Centro Universitário de Volta Redonda, Volta Redonda, RJ, Brasil.

*livia-silvaa@hotmail.com

Recebido em: 03/12/2019; Aceito em: 17/11/2020.

\section{RESUMO}

O câncer de próstata ( $\mathrm{CaP})$ é o câncer mais comum entre os homens e o segundo tipo que mais evolui para óbito nesses pacientes. Com o aumento da expectativa de vida, doenças como esta vêm sendo detectadas e tratadas precocemente, assumindo uma dimensão cada vez maior e se tornando um problema de saúde pública. Trata-se de um estudo epidemiológico descritivo, cujos dados foram disponibilizados pelo DATASUS. A população do estudo foi constituída por todos os casos de neoplasia maligna da próstata em homens de todas as idades, diagnosticados e registrados no período de 2009 a 2018 . O crescimento anual dos casos novos dessa doença foi contínuo, decrescendo somente em 2018, com uma queda de 0,34\%. Homens a partir de 50 anos englobaram o maior número dos casos, sendo as idades de 60 a 69 anos responsáveis por 38,21\% dos casos. Em brancos e pardos houve mais de $60 \%$ de taxa desta neoplasia. Observou-se uma crescente tendência de aumento da taxa de mortalidade com o aumento da idade, com homens acima de 80 anos apresentando os maiores números desse índice. $\mathrm{R} \$ 517.416 .212,44$ foram gastos nesses 10 anos com a neoplasia maligna da próstata. Concluiu-se que, no Brasil, o perfil dos pacientes com CaP é de homens acima de 50 anos, sendo que a maioria é da raça branca e se concentra no Sudeste. O número de internações e a taxa de mortalidade cresceram ao decorrer desses 10 anos analisados e a taxa de mortalidade também se mostrou maior nos idosos com CaP.

Palavras-chave: Câncer de próstata. DATASUS. Epidemiologia.

\section{ABSTRACT}

Prostate cancer (PC) is the most common cancer among men and the second type that most evolves to death in these patients. With the increase in life expectancy, diseases such as this one have been detected and treated early, taking on an increasing dimension and becoming a public health problem. This is a descriptive epidemiological study, whose data are available from DATASUS. The study population consisted of all cases of malignant prostatic neoplasm in 
men of all ages, diagnosed and recorded from 2009 to 2018. The annual growth of new cases of prostate cancer was continuous, decreasing only in 2018, with a fall of $0.34 \%$. Men aged 50 and over comprised the largest number of cases, with ages ranging from 60 to 69 years accounting for $38.21 \%$ of cases. In whites and mixed races there were more than $60 \%$ rate of this neoplasia. There was a growing trend of increasing mortality rates with the increasing age, with men over 80 showing the highest numbers in this rate. $R \$ 517,416,212.44$ were spent in these 10 years with malignant prostatic neoplasm. It is concluded that, in Brazil, the profile of PC patients is male over 50 years of age, the majority being white and concentrated in the Southeast. The number of hospitalizations and the mortality rate increased over these 10 years and the mortality rate was also higher in the elderly with PC.

Keywords: DATASUS. Epidemiology. Prostate cancer.

\section{INTRODUÇÃO}

De acordo com o Instituto Nacional de Câncer José Alencar Gomes da Silva (INCA) (2017), no Brasil, o câncer de próstata (CaP) é o mais comum entre os homens, excluindo o câncer de pele não melanoma, e é o segundo tipo que mais evolui para óbito nesses pacientes. Ele é considerado um câncer da terceira idade, já que cerca de 3/4 dos casos no mundo ocorrem após os 65 anos de idade (INCA, 2014). Além disso, a população apresentou um aumento de sua expectativa de vida, o que tornou possível uma maior detecção de casos de CaP, uma vez que ele atinge majoritariamente os idosos. Assim, essa condição patológica vem sendo detectada e tratada precocemente, tornando-se um problema de saúde pública, pois assume uma dimensão cada vez maior (SANTOS; SOUZA, 2017).

O rastreio da neoplasia maligna da próstata é possível ser realizado por meio do toque retal e da dosagem de antígeno prostático específico, porém não é recomendada pelo INCA, pois ambas as estratégias apresentam mais riscos do que possíveis benefícios no rastreamento. É indicado somente a abordagem em homens com sinais e/ou sintomas iniciais da doença (INCA, 2014).

Todavia, a busca por cuidados preventivos de saúde não é prática comum entre a população masculina, o que justifica os altos índices da doença. Normalmente, as necessidades de saúde do homem são, por ele mesmo, desvalorizadas, o que faz com que não sejam devidamente reconhecidas. Com isso, torna-se possível entender que a identidade masculina é um fator de risco para a saúde, visto que esta se torna vulnerável ao passo em que ele aceita, sem reflexão, os padrões de gênero constituídos social e culturalmente. Neste viés, entende-se que a necessidade da concretização de ações de atenção à saúde do homem, como diagnóstico, tratamento, reabilitação, manutenção e proteção, representam um desafio para o sistema público de saúde, principalmente se atreladas à reflexão sobre a influência dos valores culturais (FERNANDES et al., 2014). O objetivo desse artigo é identificar a epidemiologia dos casos de neoplasia maligna de próstata no Brasil no período de 10 anos. 


\section{MATERIAL E MÉTODOS}

Trata-se de um estudo epidemiológico descritivo, cujos dados foram obtidos por meio de consulta às seguintes bases de dados: Sistema de Informações de Mortalidade (SIM) e do Sistema de Informações Hospitalares $(\mathrm{SIH})$, respectivamente, disponibilizados pelo Departamento de Informática do Sistema Único de Saúde (DATASUS), no endereço eletrônico (http://www.datasus.gov.br), que foi acessado em 20/11/2019. O processamento e análise dos dados desenvolveram-se por meio do software Excel (Microsoft@).

A população do estudo foi constituída por todos os casos de neoplasia maligna da próstata em homens de todas as idades, diagnosticados e registrados no período de 2009 a 2018. As variáveis analisadas foram faixa etária, região, cor/raça, internação, taxa de mortalidade e valores dos serviços hospitalares. Para evitar erros de retardo de notificação, optou-se por analisar os dados disponíveis até 2018, último ano em que constavam os dados completos. Por se tratar de um banco de domínio público, não foi necessário submeter o projeto ao Comitê de Ética em Pesquisa.

\section{RESULTADOS E DISCUSSÃO}

De acordo com o World Cancer Research Fund International (2018), a neoplasia maligna de próstata está na quarta colocação da classificação dos cânceres mais prevalentes do mundo, e é o segundo mais comum em homens, precedido pelo câncer de pulmão. De acordo com Paiva, Motta e Griep (2010), a incidência do $\mathrm{CaP}$ aumenta cada dia mais. Inúmeros fatores estão associados a este fenômeno, dentre eles sobressaem-se: o aumento da expectativa de vida; campanhas frequentes para rastreamento da doença, aumentando assim o número de homens com diagnóstico de câncer; os hábitos alimentares, como alimentos de alto valor calórico e gordurosos; e as influências ambientais (PAIVA; MOTTA; GRIEP, 2010).

Atualmente, inúmeras campanhas nacionais, como o Novembro Azul, são promovidas por hospitais, sociedades médicas e outras organizações a fim de estimular o rastreamento do $\mathrm{CaP}$, apesar de inúmeras instituições estrangeiras e, no Brasil, o INCA não recomendar. O objetivo desse rastreamento seria para realizar a detecção precoce do $\mathrm{CaP}$, antes do surgimento de sintomas, o que poderia, teoricamente, aumentar a probabilidade de sucesso do tratamento, elevando a sobrevida e/ou melhorando a qualidade de vida do paciente. Contudo já foi demonstrado que esse rastreamento oferece mais riscos do que possíveis benefícios (STEFFEN et al., 2018). Por exemplo, de acordo com Bell et al. (2015), o que ocorre hoje é um sobrediagnóstico, no qual pacientes que nunca teriam apresentado sinais e sintomas dessa neoplasia são submetidos ao tratamento que podem causar efeitos adversos, como incontinência e impotência.

$\mathrm{Na}$ Tabela 1 têm-se os dados referentes ao total de número de casos de neoplasia maligna da próstata diagnosticados no período de 2009 a 2018, que totalizaram 265.483 casos novos, sendo que, destes, 31.527 ocorreram em 2017, representando $11,88 \%$ do número total de casos registrados ao longo de 10 anos. O crescimento anual foi contínuo, decrescendo somente no ano de 2018, 
apresentando uma queda apenas de $9,65 \%$ de novos casos comparando-se ao ano anterior.

Tabela 1 - Distribuição total de número de casos de neoplasia maligna da próstata diagnosticados em 10 anos, segundo ano de internação

\begin{tabular}{lcc} 
Ano de internação & № & $\%$ \\
\hline 2009 & 19.463 & $7,33 \%$ \\
2010 & 21.676 & $8,16 \%$ \\
2011 & 23.846 & $8,98 \%$ \\
2012 & 25.394 & $9,57 \%$ \\
2013 & 26.350 & $9,93 \%$ \\
2014 & 27.377 & $10,31 \%$ \\
2015 & 29.459 & $11,10 \%$ \\
2016 & 29.769 & $11,21 \%$ \\
2017 & 31.527 & $11,88 \%$ \\
2018 & 30.622 & $11,53 \%$ \\
\hline
\end{tabular}

Total: 265.483

Fonte: Ministério da Saúde - Sistema de Informações Hospitalares do SUS (SIH/SUS).

Observa-se na Tabela 2 que, em relação à faixa etária, homens a partir de 50 anos englobam o maior número dos casos de neoplasia maligna em 10 anos, sendo que as idades de 60 a 69 anos foram responsáveis pela maioria, com $101.430(38,21 \%)$.

De acordo com Bell et al. (2015), casos de neoplasia maligna da próstata são achados em alguns homens muito jovens e a prevalência desse câncer aumenta em ritmo crescente com a idade, sugerindo que este câncer é geralmente uma doença de desenvolvimento lento e com uma longa fase préclínica. O desenvolvimento dos sintomas e o diagnóstico clínico ocorrem principalmente em homens mais velhos, sendo que muitos morrem de outras causas muito antes de qualquer sintoma ser clinicamente manifestado (BELL et al., 2015).

Tabela 2 - Distribuição total de número de casos de neoplasia maligna da próstata diagnosticados em 10 anos, segundo a faixa etária

\begin{tabular}{lcc} 
Faixa Etária & № & $\%$ \\
\hline Abaixo de 19 anos & 368 & $0,14 \%$ \\
$20-29$ anos & 181 & $0,07 \%$ \\
$30-39$ anos & 318 & $0,12 \%$ \\
$40-49$ anos & 4.515 & $1,70 \%$ \\
$50-59$ anos & 38.501 & $14,50 \%$ \\
$60-69$ anos & 101.430 & $38,21 \%$ \\
$70-79$ anos & 84.395 & $31,79 \%$ \\
Acima de 80 anos & 35.775 & $13,8 \%$ \\
\hline
\end{tabular}

Fonte: Ministério da Saúde - SIH/SUS.

Quanto a cor/raça, segundo o DATASUS, os brancos e os pardos alcançaram juntos mais de $70 \%$ dos casos de neoplasia maligna da próstata em 10 anos, com 102.638 (38,66\%) e 89.519 (33,72\%), respectivamente (Tabela 3). 
Contudo, em oposição ao resultado apresentado pelo DATASUS, de acordo com a Sociedade Brasileira de Urologia (2018), o CaP em homens negros apresenta uma maior incidência do que em brancos.

Na população norte-americana, já é bem estabelecida uma considerável diferença da incidência e mortalidade do $\mathrm{CaP}$ entre homens da raça negra e branca, sendo 3 e 2,4 vezes maiores em homens negros, respectivamente (PERNAR et al., 2018). Essa discrepância entre as raças tem sido correlacionada com baixo status socioeconômico e diagnóstico em estágios avançados devido à dificuldade, dessa população, de acesso aos serviços de saúde (BENJAMINS et al., 2016). Além disso, foi demonstrado em um estudo de coorte realizado nos EUA, que homens negros com neoplasia maligna da próstata não-metastático, quando no mesmo estágio da doença, pareciam ter mortalidade comparável aos homens brancos quando ambos possuíam acesso aos serviços de saúde e ao tratamento padrão (DESS et al., 2019).

Já no estudo epidemiológico realizado por Tourinho-Barbosa, Pompeo e Glina (2016), no qual foram analisados somente artigos que contemplavam a população do Brasil e da América-Latina, os dados referentes à prevalência do $\mathrm{CaP}$ por raça foram conflitantes, sendo que alguns estudos demonstraram uma prevalência mais alta na população negra e outros não obtiveram uma diferença significativa. É necessário um estudo mais aprofundado para explicar as causas dessas disparidades.

Tabela 3 - Distribuição total de número de casos de neoplasia maligna da próstata diagnosticados por ano, segundo a cor/raça

\begin{tabular}{lcc}
\hline Cor/raça & № & $\%$ \\
\hline Branca & 102.638 & $38,66 \%$ \\
Preta & 19.430 & $7,32 \%$ \\
Parda & 89.519 & $33,72 \%$ \\
Amarela & 3.104 & $1,17 \%$ \\
Indígena & 101 & $0,04 \%$ \\
Sem informação & 50.691 & $19,09 \%$
\end{tabular}

Fonte: Ministério da Saúde - SIH/SUS.

Quanto à distribuição total de número de casos, segundo a região (Tabela 4), os dados oferecidos pelo DATASUS não apresentaram um padrão bem definido. O ideal seria o DATASUS apresentar os números de casos por habitantes, além da distribuição total de número de casos de CaP, para melhor comparação com dados de outras fontes. De acordo com Santos (2018), as regiões brasileiras mais desenvolvidas (Sul, Sudeste e Centro-Oeste) predominam, em homens, o CaP junto aos cânceres colorretal e pulmonar, assemelhando-se aos países mais desenvolvidos. Já nas regiões menos desenvolvidas (Norte e Nordeste), o CaP também está entre os mais incidentes junto ao câncer de estômago, dados semelhantes aos de países menos desenvolvidos. Esse perfil é um reflexo das desigualdades regionais no Brasil, que abrange desde as diferenças na expectativa de vida, condições socioeconômicas, até o acesso aos serviços de saúde para diagnóstico oportuno e tratamento adequado (SANTOS, 2018). 
Tabela 4 - Distribuição total de número de casos de neoplasia maligna da próstata diagnosticados em 10 anos, segundo a região

\begin{tabular}{lcc} 
Região & № & $\%$ \\
\hline Norte & 7.448 & $2,81 \%$ \\
Nordeste & 58.950 & $22,20 \%$ \\
Sudeste & 138.659 & $52,23 \%$ \\
Sul & 43.060 & $16,22 \%$ \\
Centro-oeste & 17.366 & $6,54 \%$ \\
\hline
\end{tabular}

Fonte: Ministério da Saúde - SIH/SUS.

$\mathrm{Na}$ Tabela 5, têm-se os dados referentes à distribuição total da taxa de mortalidade dos casos de neoplasia maligna da próstata diagnosticados segundo o ano de internação e idade. Observa-se uma crescente tendência de aumento da taxa de mortalidade com o aumento da idade, com homens acima de 80 anos apresentando os maiores números desse índice em todos os anos. Entretanto, a taxa de mortalidade não diminui ao decorrer dos 10 anos, pelo contrário, apresentou um discreto aumento, com exceção nas idades entre 20 29 e 40 - 49, nas quais houve uma discreta diminuição.

Como já citado antes, o CaP costuma apresentar sintomas clínicos somente em homens mais velhos, já que essa neoplasia apresenta um gradual e progressivo crescimento; com isso, em idades mais avançadas, tende a estar em uma fase de desenvolvimento mais tardio, justificando as maiores taxas de mortalidade acima dos 80 anos. E quanto ao aumento da taxa de mortalidade, parece estar relacionado com o aumento da expectativa de vida, com o maior acesso ao cuidado em saúde, assim com o aumento da documentação de casos, e, por último, com o fato da mudança do estilo de vida, incluindo inatividade física, obesidade e fatores dietéticos (RAWLA, 2019).

Tabela 5 - Distribuição total da taxa de mortalidade dos casos de neoplasia maligna da próstata diagnosticados, segundo o ano de internação e idade

\begin{tabular}{lcccccccc}
\hline $\begin{array}{l}\text { Ano de } \\
\text { internação }\end{array}$ & $\begin{array}{c}\text { Abaixo } \\
\text { de 19 } \\
\text { anos }\end{array}$ & $\mathbf{2 0 - 2 9}$ & $\mathbf{3 0 - 3 9}$ & $\mathbf{4 0 - 4 9}$ & $\mathbf{5 0 - 5 9}$ & $\mathbf{6 0 - 6 9}$ & $\mathbf{7 0 - 7 9}$ & $\begin{array}{c}\text { Acima } \\
\text { de 80 } \\
\text { anos }\end{array}$ \\
\hline 2009 & 9,52 & 25,00 & 5,00 & 3,67 & 3,40 & 5,59 & 10,34 & 17,46 \\
2010 & - & 15,00 & 5,88 & 3,54 & 4,11 & 5,00 & 10,04 & 17,43 \\
2011 & 1,17 & 8,00 & 2,94 & 2,12 & 4,86 & 5,35 & 9,75 & 18,96 \\
2012 & 11,64 & - & 8,11 & 4,75 & 4,21 & 5,04 & 9,92 & 17,85 \\
2013 & 7,77 & 5,88 & 13,04 & 4,15 & 4,58 & 5,32 & 10,62 & 19,12 \\
2014 & - & 9,52 & 3,33 & 4,67 & 4,41 & 5,52 & 11,43 & 19,70 \\
2015 & 11,5 & 15,38 & 8,00 & 5,05 & 4,05 & 5,19 & 11,13 & 21,82 \\
2016 & 7,57 & 5,26 & 12,12 & 4,13 & 3,95 & 6,59 & 10,69 & 20,56 \\
2017 & 10,00 & 11,76 & 7,41 & 4,12 & 4,84 & 6,26 & 10,74 & 21,85 \\
2018 & 20,00 & - & 5,71 & 2,87 & 4,45 & 5,66 & 11,17 & 21,81 \\
\hline
\end{tabular}

Fonte: Ministério da Saúde - SIH/SUS.

Observa-se na Tabela 6 que, em relação ao total dos valores dos serviços hospitalares gastos, $\mathrm{R} \$ 515.650 .149,74$ foram gastos nesses 10 anos, mostrando a sobrecarga financeira no sistema de saúde que o $\mathrm{CaP}$ causa no Brasil. Esse fato pode ser explicado pelo aumento nas taxas de incidência de 
$\mathrm{CaP}$ ao longo dos anos e os principais fatores para isso são o aumento da expectativa de vida da população e o rastreamento indevidamente realizado, culminando no sobrediagnóstico e no tratamento desnecessário de pacientes que nunca teriam apresentado sinais e sintomas dessa neoplasia futuramente (INCA, 2017).

Tabela 6 - Distribuição total dos valores dos serviços hospitalares dos casos de neoplasia maligna da próstata em 10 anos, segundo regime do hospital

\begin{tabular}{lcc}
\hline & \multicolumn{2}{c}{ Valores dos serviços hospitalares } \\
\hline Regime & No & $\%$ \\
\hline Público & $72.756 .092,10$ & $14,11 \%$ \\
Privado & $211.669 .436,12$ & $41,05 \%$ \\
Ignorado & $231.224 .621,52$ & $44,84 \%$ \\
\hline
\end{tabular}

Total: $515.650 .149,74$

Fonte: Ministério da Saúde - SIH/SUS.

Em relação às limitações do presente estudo, aponta-se que o uso de dados secundários não permite ao pesquisador controlar possíveis erros decorrentes de digitação e de registro, além de possíveis subnotificações. Apesar disto, acredita-se que, por se tratar de dados nacionais oficiais e de preenchimento obrigatório em todos os serviços de saúde, seus resultados permitiram o alcance dos objetivos propostos.

\section{CONCLUSÃO}

Por meio do presente estudo, podemos concluir que, no Brasil, o perfil dos pacientes com CaP são homens acima de 50 anos idade, entre 60 e 69 anos, principalmente, sendo a maioria da raça branca. Mais de $50 \%$ desses homens se concentram na região Sudeste e tanto o número de internações quanto a taxa de mortalidade cresceram ao decorrer desses 10 anos analisados. A taxa de mortalidade também mostrou uma maior da taxa de mortalidade nos idosos com CaP. $\mathrm{R} \$ 517.416 .212,44$ foram gastos nesses 10 anos.

Esses dados fornecidos pelo DATASUS podem ser uma ferramenta útil para os profissionais de saúde repensarem a sua prática e direcionarem investimentos nesta área do conhecimento, além de oportunizarem, aos gestores públicos, o planejamento de estratégias preventivas específicas a esta população. Assim, conhecer a epidemiologia desta doença é essencial para 0 direcionamento das ações de promoção, prevenção e reabilitação.

\section{REFERÊNCIAS}

BRASIL. Ministério da Saúde. Departamento de Informática do Sistema Único de Saúde (DATASUS). Brasília. 2008. Disponível em: http:// www.datasus.gov.br. Acesso em: 20 de nov. de 2019.

BELL, K. J. L. et al. Prevalence of incidental prostate cancer: A systematic review of autopsy studies. International Journal of Cancer, v. 137, n. 7, p. 1749-1757, 2015. 
BENJAMINS, M. R. et al. Racial Disparities in Prostate Cancer Mortality in the 50 Largest US Cities. Cancer Epidemiology, v. 44, p. 125-131, 2016.

DESS, R. T. et al. Association of Black Race with Prostate Cancer-Specific and Other-Cause Mortality. JAMA Oncology, v. 5, n. 7, p. 975-983, 2019.

FERNANDES, M. V. et al. P. Perfil epidemiológico do homem com câncer de próstata atendido em um hospital universitário. Revista Cogitare Enfermagem, v. 19, n. 2, p. 333-340, 2014.

INSTITUTO NACIONAL DE CÂNCER JOSÉ ALENCAR GOMES DA SILVA. Monitoramento das ações de controle do Câncer de Próstata. Rio de Janeiro, RJ, 2014.

INSTITUTO NACIONAL DE CÂNCER JOSÉ ALENCAR GOMES DA SILVA. Monitoramento das ações de controle do Câncer de Próstata. Rio de Janeiro, RJ, 2017.

INSTITUTO NACIONAL DE CÂNCER JOSÉ ALENCAR GOMES DA SILVA. Estimativa 2020: Incidência de Câncer no Brasil. Rio de Janeiro, RJ, 2019.

SOCIEDADE BRASILEIRA DE UROLOGIA. Novembro Azul orienta sobre o câncer de próstata. 11 de out. de 2018. Disponível em: <https://portaldaurologia.org.br/novembro-azul/novembro-azul-orienta-sobre-ocancer-de-prostata/\# >. Acesso em: 07 de ago. de 2020.

PAIVA, E. P.; MOTTA, M. C. S.; GRIEP, R. H. Conhecimentos, atitudes e práticas acerca da detecção do câncer de próstata. Acta Paulista de Enfermagem, v. 23, n. 1, p. 88-93, 2010.

PERNAR, C. H., et al. The Epidemiology of Prostate Cancer. Cold Spring Harbor Laboratory Press, v. 8, n. 12, a030361, 2018.

RAWLA, P. Epidemiology of Prostate Cancer. World Journal of Oncology, v. 10, n. 2, p. 63-89, 2019.

SANTOS, J. P.; SOUZA, A. P. Considerações sobre o Câncer de Próstata: Revisão de Literatura. Revista Multidisciplinar e de Psicologia, v. 10, n. 33, p. 100-115, 2017.

SANTOS, M. O. Estimate 2018: Cancer Incidence in Brazil. Revista Brasileira de Cancerologia, v. 66, n. 1, p. 119-120, 2018.

STEFFEN, R. E. et al. Rastreamento populacional para o câncer de próstata: mais riscos que benefícios. Revista de Saúde Coletiva, v. 28, n. 2, e280209, 2018. 
TOURINHO-BARBOSA, R. R.; POMPEO, A. C. L.; GLINA, S. Prostate cancer in Brazil and Latin America: epidemiology and screening. International Brazilian Journal of Urology, v. 42, n. 6, p. 1081-1090, 2016.

WORLD CANCER RESEARCH FUND. Prostate cancer statistics. 2018. Disponível em: < https://www.wcrf.org/dietandcancer/cancer-trends/prostatecancer-statistics >. Acesso em: 07 de ago. de 2020. 